\title{
A polyethylene glycol-based method for extraction of extracellular vesicles from Lactobacillus casei as vaccine delivery vehicle
}

\author{
Ebrahimi Vargoorani $\mathrm{M}^{1}$, Modarressi $\mathrm{MH}^{2}$, Motevaseli $\mathrm{E}^{3}$, Vaziri $\mathrm{F}^{4,5}$, Siadat $\mathrm{SD}^{4,5^{*}}$ \\ ${ }^{1}$ Department of Biology, Science and Research Branch, Islamic Azad University, Tehran, Iran. \\ ${ }^{2}$ Department of Medical Genetics, School of Medicine, Tehran University of Medical Sciences, Tehran, Iran. \\ ${ }^{3}$ Department of Molecular Medicine, School of Advanced Technologies in Medicine, Tehran University of Medical Sciences, \\ Tehran, Iran. \\ ${ }^{4}$ Department of Mycobacteriology and Pulmonary Research, Pasteur Institute of Iran, Tehran, Iran. \\ ${ }^{5}$ Microbiology Research Center, Pasteur Institute of Iran, Tehran, Iran.
}

\begin{abstract}
Introduction The secretion of extracellular vesicles (EVs) has been neglected in Gram-positive bacteria due to the absence of an outer membrane and the difficulties of proper visualization. Here we aimed to prove that lactobacillus casei can secrete extracellular vesicles. Methods: EVs were extracted from Lactobacillus casei, cultured in De Man, Rogosa and Sharpe broth, using a polyethylene glycol (PEG) solution. The characteristics of the EVs were analyzed by electron microscopy, Dynamic Light Scattering (DLS) and SDS-PAGE. Results: The electron microscopy showed rounded vesicles with average diameter of $300 \mathrm{~nm}$. The protein content of this nanostructure was $2.5 \mathrm{mg} / \mathrm{ml}$ with a protein pattern within the range of 10-200 $\mathrm{kDa}$. DLS result showed populations of approximately $300 \mathrm{~nm}$ while the extracted EVs had a negative zeta potential. Conclusion: A new method of producing functional molecules from probiotic bacteria was presented. Our results indicated EVs purity with acceptable conformation. Further investigations are necessary to elucidate the efficacy, practicality and mechanism of action of such EVs in clinical practices, especially for development of bio-compounds and vaccine delivery vehicles.
\end{abstract}

KEYWORDS: Lactobacillus casei, extracellular vesicles; polyethylene glycol.

\section{INTRODUCTION}

Probiotics possess health promoting properties. One of the most important probiotic strains belongs to Lactobacillus genus. Among Lactobacillus species, Lactobacillus casei is one of the most common probiotics with industrial applications. It has been reported to have positive effects on human health such as causing remarkable improvements in immunity [1, 2], allergies [3] and cholesterol levels [3]. Over the past few years, scientists have proven that probiotics and their bio-compound can have many benefits. For instance, they can inhibit human immunodeficiency virus (HIV) and herpes simplex virus (HSV) infections [4]. Moreover, components from Lactobacillus spp. can down-regulate the proinflammatory signaling pathways [5]. Also, exopolysaccharides of Lactobacilli have anti-elastase and anti-collagenase potentials on human fibroblast [6].

Interestingly, the probiotic lactobacilli used in the food industry

*Corresponding Author: Seyed Davar Siadat, Department of Mycobacteriology \& Pulmonary Research. Microbiology Research Center (MRC), Pasteur Institute of Iran, Tehran, Iran.

Email: d.siadat@gmail.com

Tel/Fax: (+98) 2164112213/ (+98) 2164112823 could the best option to treat many infectious diseases. In fact, one of the properties of lactobacilli is their competition with the pathogens for the cell attachments [7]; as it has been confirmed that lactobacilli have anti-adhesive effects on certain pathogenic bacteria[8].

Lactic acid bacteria, are widely used in the food industry; therefore, the recognition of molecules derived from lactic acid bacteria can be useful to develop a new generation of functional compounds. One of the molecules secreted by bacteria is extracellular vesicles (EV)[9] which contain lipid bilayers that within 20-500 $\mathrm{nm}$ range in diameter. These structures do usually contain varied cargos, such as lipoproteins, nucleic acids, toxins and communication signals which are of great importance for the microbial pathogenesis and physiology [10]. Moreover, we have demonstrated that EVs can also be used as a delivery system [11]. It has been shown that E. coli EVs could be used as a vaccine vehicle system; however, due to presence of lipopolysaccharides in these nanostructures, a number of structural changes are needed [12].

Despite their great significance, the processes of EV isolation and characterization are still quite challenging and it would be essential to characterize an optimal method for this purpose. EV 
purification has been accomplished by at least four different methods, namely immunoaffinity [13], chromatography [14], ultracentrifugation and polymer-based precipitation [15]. Among these methods, ultracentrifugation is the most common one. The major advantage of ultracentrifugation is that it can manage large sample volumes for an improved throughput. Nevertheless, EV isolation by this method is time-consuming and a low yield of a degraded product is generally obtained. Various commercially prepared isolation kits have been recently offered; however their applications are limited due to their high cost and relatively low purity of the preparations [16].

The isolation of probiotic component is an important research priority and challenge for the food as well as the vaccine industries and other science sectors. In absence of a standard isolation technique for $L$. casei which has the benefits of lacking lipopolysaccharides in its structure and also being regarded as a safe probiotic bacterium, here we investigated a method for its EV purification using polyethylene glycol (PEG). Using this technique for the first time, we were able to isolate EVs from large volumes of media, rapidly and costeffectively via low-speed centrifugations.

\section{MATERIALS and METHODS}

\section{Bacterial strain and culture method}

L. casei (ATCC 393 strains) was obtained from Iranian Biological Resource Center (Tehran, Iran) and was cultured in De Man, Rogosa and Sharpe agar (MRS; Sigma-Aldrich; Germany). In order to obtain more biomasses, a large volume of the bacterial culture was prepared $\left(37^{\circ} \mathrm{C}, 24 \mathrm{~h}\right)$ in MRS broth [17].

Preparation of PEG solution for isolation of EVs

PEG solution is usually applied to concentrate virus particles. A virus enrichment method was modified to concentrate and isolate EVs of similar size and biochemical features. PEG (Sigma, 81260, Germany) with average Mw of 6000 Da was mixed with sodium chloride $(1 \mathrm{M})$ and filtered water (ELGA Purelab Flex Purification System; 18.3 MegaOhm System, Germany). Afterwards, the stock solution was added to the bacterial culture supernatant of the same volume. Concentrations at $16 \%$ of PEG solution were evaluated in terms of ability to recover $20-1000 \mathrm{~nm}$ particles. In precipitation of the solutions, the concentration of sodium chloride was kept at $1 \mathrm{M}[16]$.

\section{Extraction of the EVs}

A large L. casei bacterial culture $\left(37^{\circ} \mathrm{C}\right)$ in MRS broth was prepared overnight in order to obtain more biomasses. From the bacterial culture, the vesicle-containing medium was centrifuged for $45 \mathrm{~min}$ at $6000 \times \mathrm{g}$. Centrifugation was then performed again at $10000 \times \mathrm{g}$ for $30 \mathrm{~min}\left(4^{\circ} \mathrm{C}\right)$ to remove the cellular debris. After centrifugation, the medium was added at $4^{\circ} \mathrm{C}$ to an equal volume of $16 \%$ PEG solution. The sample was then completely mixed via inversion and was incubated overnight for a minimum of $12 \mathrm{~h}$ at $4{ }^{\circ} \mathrm{C}$. The samples were centrifuged the following day, using a tabletop centrifuge device at $3200 \times \mathrm{g}$ (Eppendorf; model $5810 \mathrm{R}$ with an S-4-104 swing bucket rotor; $3,214 \mathrm{~g}$; Germany) for $1 \mathrm{~h}$ at $4^{\circ} \mathrm{C}$. After the conical tubes were decanted, they were allowed to drain for 5 min, with occasional tapping to discard excess PEG [16]. Finally, particle-free sucrose was used to resuspend the samples. For further analyses, the sample subsets were stored at $-80^{\circ} \mathrm{C}$.

\section{SDS-PAGE}

Samples were loaded on $12 \%$ gel to determine the protein patterns following electrophoresis. The protein concentrations were measured using Nanodrop and Bradford assays, based on spectrophotometric methods.

\section{Scanning Electron Microscopy (SEM) analysis}

SEM was performed to determine the morphology and size of the EVs. The samples were placed in aluminum holders and then sputter coated using $5 \mathrm{~nm}$ of gold. The prepared samples were examined by SEM (KYKY Technology, China) [18].

Evaluation of EVs size via dynamic light scattering (DLS) and Zeta potential measurements

The size distribution and diameter of EVs which were resuspended in sucrose solution, were determined using DLS with a particle size analyzer (90Plus/BI-MAS Multi-Angle Analyzer, Brookhaven Instruments Corp, UK) [19]. A zeta potential analyzer (ZetaPlus; Brookhaven Instruments Corp, UK) was used to determine the zeta potential of EVs in $3 \%$ sucrose solution [19].

\section{RESULTS}

\section{Evaluation of EVs by SDS-PAGE}

Total protein concentrations of the EVs were analyzed by NanoDrop and Bradford assays. The evaluated concentrations of the purified total protein were $2.5 \mathrm{mg} / \mathrm{ml}$ and $2.31 \mathrm{mg} / \mathrm{ml}$ by Bradford and Nanodrop methods, respectively. The protein profile of the isolated EVs was in the range of 10-200 $\mathrm{kDa}$ as observed by SDS-PAGE method (Fig.1).

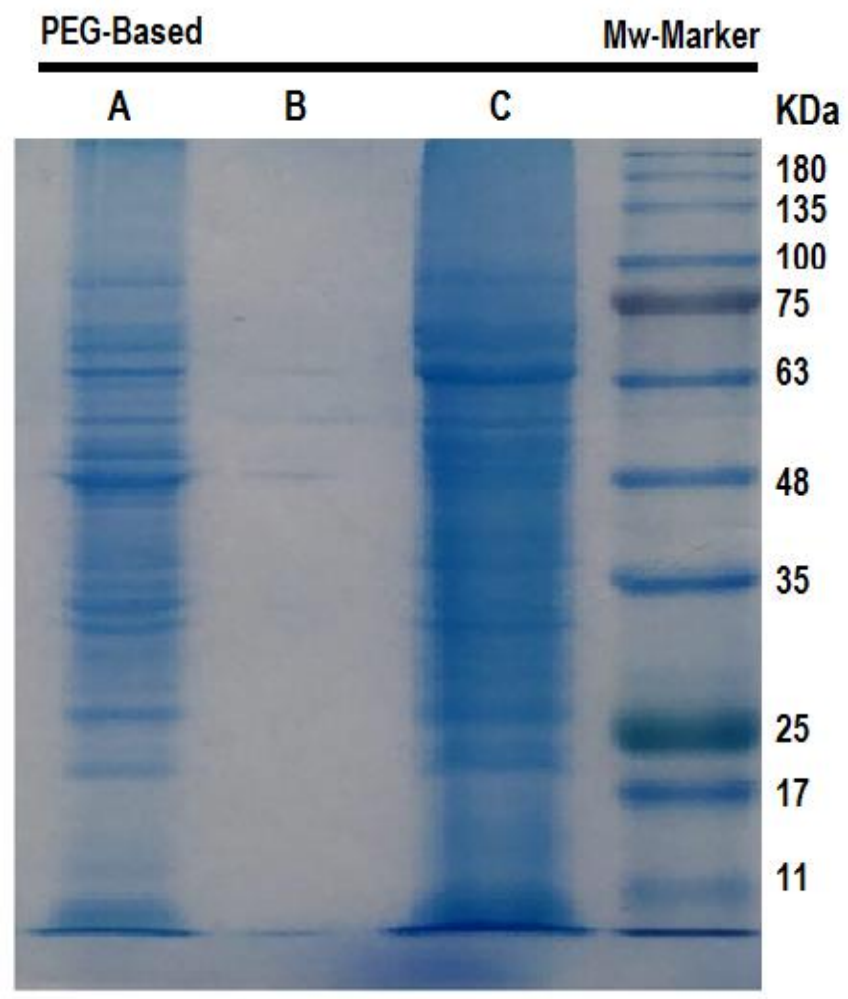

Fig.1. SDS-PAGE of EVs purified from L. casei, stained by Coomassie method. SDS-PAGE of $L$. casei derived EVs by ultracentrifugation (C lane) and PEGbased method (A lane) and B lane: empty. The ladder shows the protein bands between 11-180 $\mathrm{kDa}$. 
The electron microscopy showed spherical vesicles with specific physicochemical properties with diameters ranged between 200-400 nm (Fig. 2).

\section{Physical characterization of the $L$. casei vesicles}

EVs preparations from L. casei cultures were evaluated by DLS technique to determine the size distribution of the EVs. The DLS analysis showed 2 populations of approximately 50-500 $\mathrm{nm}$ in preparations from the PEG-based method (Fig. 3).
Activity decreased significantly by $45.85 \%$ after EDC-NS3 immunization but CDC-NS3 immunization caused a nonsignificant decrease $(3.14 \%)$ in its activity.

\section{Zeta Potential Measurements}

Zeta potential of the isolated EVs was determined for two purification techniques. Based on our data, the measurements of the zeta potential of the vesicles were negative $(-11 \mathrm{mV})$, as shown in Fig. 4.

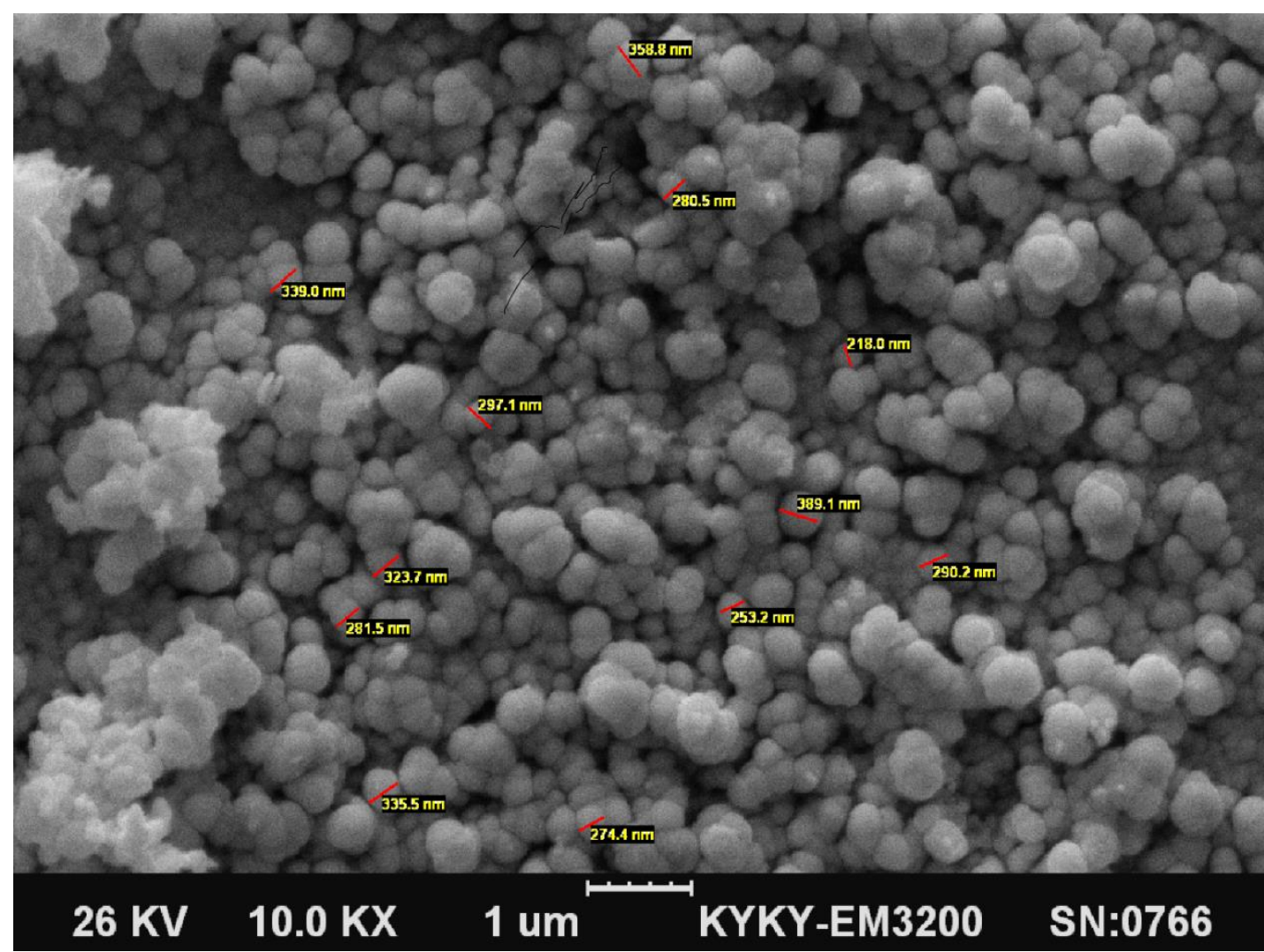

Fig.2. SEM micrographs of the EVs preparations by PEG solution (magnification: $\times 10 \mathrm{~K}$ ).

Size Distribution by Intensity

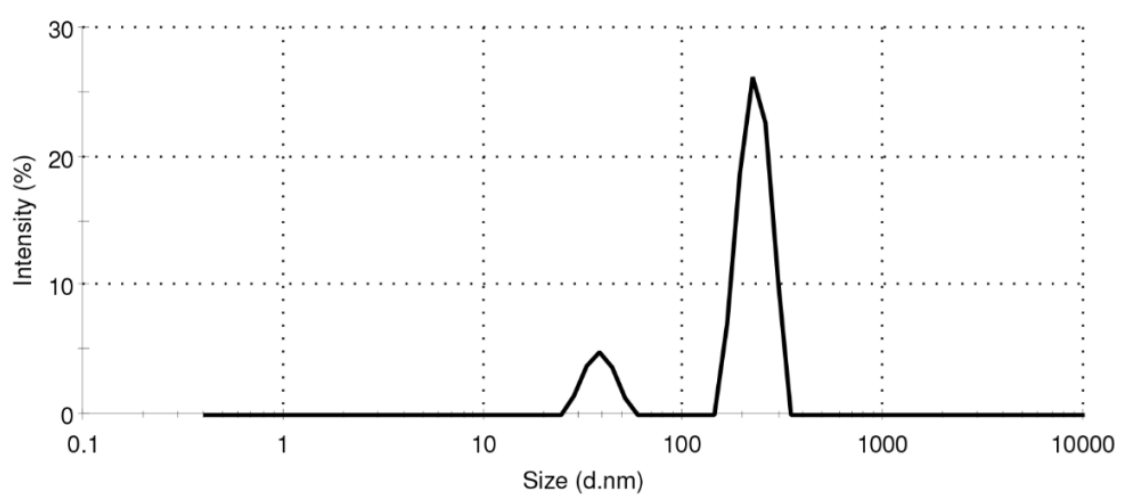

Fig. 3. Size distribution report by intensity of the EVs from the PEG-based solution. 


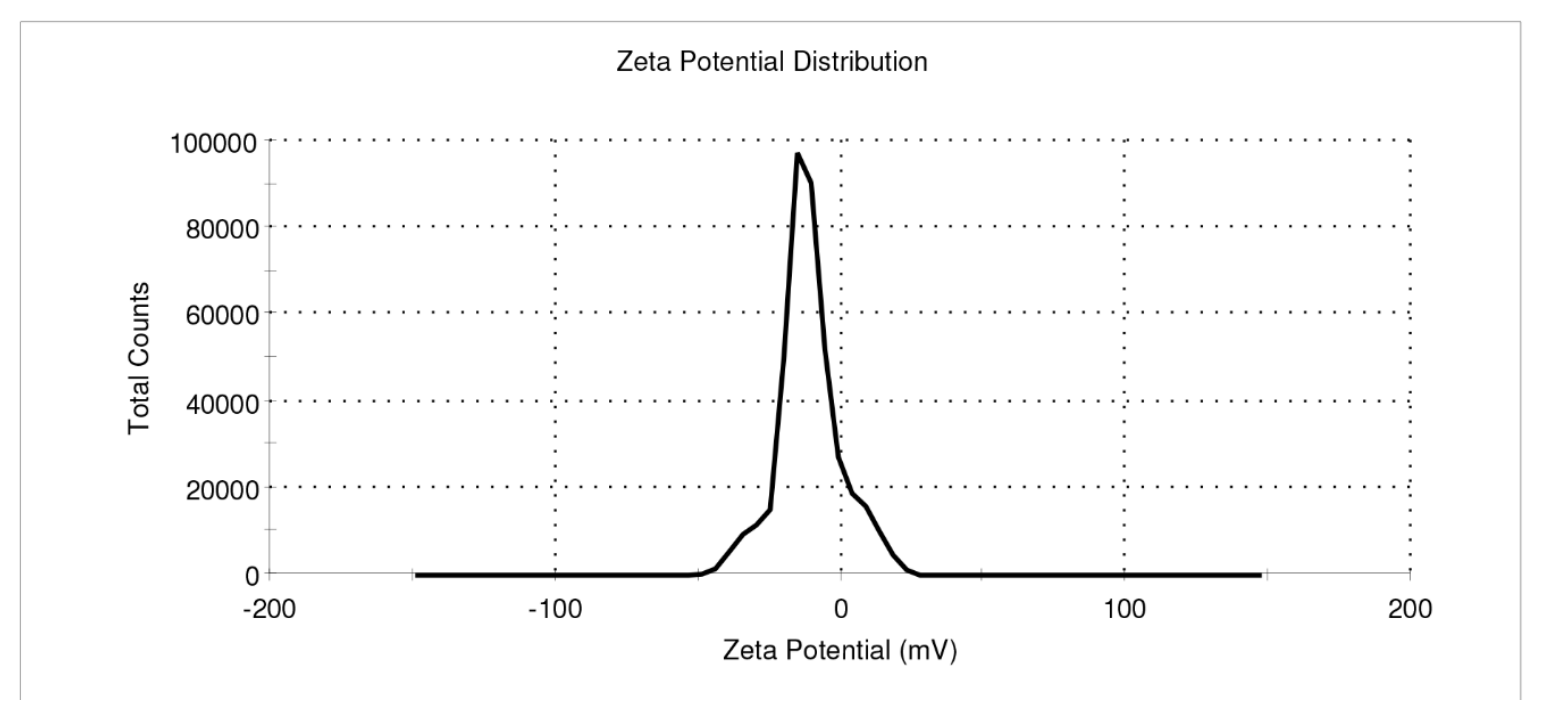

Fig. 4. Zeta potential report of the EVs extracted by the PEG-based solution.

\section{DISCUSSION}

Due to the importance of probiotics in basic scientific studies and commercial applications, we attempted to study a new method of EVs from a member of the probiotic bacteria using a PEG-based solution. Besides increasing food productions based on probiotics, a large number of publications have shown that probiotic bacteria have significant positive effects on human health. For example, it has been shown that components from Lactobacillus spp. can down-regulate the proinflammatory signaling pathways [5]. Moreover, derivatives from probiotics like EVs have opened the door for development of a new generation of functional compounds, such as vaccine vehicles. In this study, we demonstrated that L. casei which is one of the most important probiotics, is able to produce and release EVs. Many methods have been developed for isolation and characterization of EVs in recent years and it could be assumed that any of these methods could have direct effects on their downstream analyses. Among many different techniques to identify EVs [20-22], here we used five of them.

Observations by electron microscopy are among the most common techniques for detecting extracellular vesicles in recent years [23]. For instance, the structure and size of Staphylococcus aureus derived EVs have been studied using Transmission Electron Microscopy (TEM) [24]. Moreover, Li et al. (2017) have isolated EVs from Lactobacillus plantarum and have shown by electron microscopy that the size of these nanostructures is $30-300 \mathrm{~nm}$. According to many studies, the diameter of EVs from Gram-positive organisms are 20-200 $\mathrm{nm}$, which is similar to the size of EVs from Gram-negative bacteria [10]. It is noteworthy that the size of EVs from Grampositive bacteria could be varied. This feature indicates that the synthesis of EVs is regulated in a variety of ways, although vesiculogenesis is considered a general procedure. We performed SEM in this study to determine the spatial structure and size of these nanostructures which had an average diameter between $200-400 \mathrm{~nm}$.

Our evaluations of total protein concentrations and profile of $L$. casei derived-EV were based on similar methods used by other researchers $[23,25]$. Our data indicated that the concentration of the EVs by the PEG-based method was approximately 2.5 $\mathrm{mg} / \mathrm{ml}$. Moreover, the Mw of $L$. casei derived-EVs ranged from 10 to $200 \mathrm{kDa}$, confirmed by SDS-PAGE.

The physical characterizations of L. case $i$ vesicles were done by DLS analysis and zeta potential measurements. DLS analysis by Brown et al. has revealed two populations of approximately 50 and $150-250 \mathrm{~nm}$ in EVs preparations from Bacillus subtilis strain 168 [26]. After isolating EVs from L. casei by the PEGbased method, we noted that the isolated vesicles produced different DLS profiles. Our DLS analysis showed heterogeneous diameters, in consistence with the sizes predicted by the electron microscopic studies; nonetheless, this technique overestimates or underrates the diameter of the vesicles. The dimensions reported in DLS analysis were consistent with the SEM measurements.

EVs extracted by the PEG-based method were negatively charged according to zeta potential analyses and had a high zeta potential. The small zeta potentials of the EVs show their instability in solution and indicate the need for caution in storing and handling EVs at temperatures other than $-80^{\circ} \mathrm{C}$. These results were similar to the extracellular vesicles described by Dean et al.[27]. When the potential is from 0 to \pm 5 , we call it small, and in this situation, attractive forces greater than repulsion and dispersion are disrupted and flocculation happens. Therefore, EVs with low zeta potential (negative or positive) are electrically unstable and tend to coagulate. Therefore, the extraction methods can affect the zeta potential and the stability of the EVs.

Ultracentrifugation is still the standard method for EV isolation, however, it requires parameters which are difficult to optimize, including the g-force, the rotor type (fixed angle or swinging bucket), and centrifugal radius. Moreover, it is relatively timeconsuming and sensitive and requires access to an ultracentrifuge apparatus and prior trainings. The EVs integrity may also be impaired following prolonged ultracentrifugation at high speeds [28] and some observations have suggested that the ultracentrifuge method produces vesicles with reduced biological activities [29]. On the other hand, the use of commercial kits is not cost-effective.

In conclusion, our results proved that EVs purity and conformation were within an acceptable range and by this method EVs can be easily and inexpensively isolated from 
probiotic bacteria using a low-speed centrifugation. In addition, the possibility to recover many vesicles with higher resistance makes this method advantageous. Since EVs can deliver bacterial products to the host, extensive efforts are being made to deliver immunogenic antigens by these structures. The positive effects of vaccination with EVs of Gram-positive bacteria have been carried out against Clostridium perfringens, $S$. pneumoniae, $B$. anthracis, $M$. tuberculosis, and $S$. aureus. Based on animal models, the above mentioned EVs vaccinations have prolonged the survival time upon lethal challenges [30-33]. The identification of active components of L.casei secreted EVs and its potential therapeutic use remain to be investigated. Further researches are necessary to elucidate the efficacy, the practicality and the mechanisms of action of such EVs in clinical practices, especially as vaccine delivery vehicles.

\section{ACKNOWLEDGEMENT}

We thank Prof. Andrea Masotti for his comments and editorial support. We are also grateful to our colleagues at Mycobacteriology and Pulmonary Research Department and Microbiology Research Center of Pasteur Institute of Iran. This research received funding from the National Institute for Medical Research Development (Project No942995) and Pasteur Institute of Iran.

\section{CONFLICT OF INTEREST}

None of the authors had any personal or financial conflict of interest.

\section{REFERENCES}

1. Galdeano CM, Perdigon G. The probiotic bacterium Lactobacillus casei induces activation of the gut mucosal immune system through innate immunity. Clinical and Vaccine Immunology. 2006;13(2):219-26. doi:doi: 10.1128/CVI.13.2.219-226.2006.

2. Kim S-N, Lee WM, Park KS, Kim JB, Han DJ, Bae J. The effect of Lactobacillus casei extract on cervical cancer cell lines. Contemporary Oncology. 2015;19(4):306. doi:doi: 10.5114/wo.2014.45292.

3. Kumar A, Kumar M, Ghosh M, Ganguli A. Modeling in vitro cholesterol reduction in relation to growth of probiotic Lactobacillus casei. Microbiology and immunology. 2013;57(2):100-10. doi:doi.org/10.1111/1348-0421.12008.

4. Zabihollahi R, Motevaseli E, Sadat SM, Azizi-Saraji AR, Asaadi-Dalaie S, Modarressi MH. Inhibition of HIV and HSV infection by vaginal lactobacilli in vitro and in vivo. DARU Journal of Pharmaceutical Sciences. 2012;20(1):53. doi:doi.org/10.1186/2008-2231-20-53.

5. Villena J, Kitazawa H. Modulation of intestinal TLR4inflammatory signaling pathways by probiotic microorganisms: lessons learned from Lactobacillus jensenii TL2937. Frontiers in immunology. 2014;4:512. doi:doi.org/10.3389/fimmu.2013.00512.

6. Shirzad M, Hamedi J, Motevaseli E, Modarressi MH. Anti-elastase and anti-collagenase potential of Lactobacilli exopolysaccharides on human fibroblast. Artificial cells, nanomedicine, and biotechnology. 2018:1-11. doi:doi.org/10.1080/21691401.2018.1443274.

7. Kasra-Kermanshahi R, Mobarak-Qamsari E. Inhibition effect of lactic acid bacteria against food born pathogen, Listeria monocytogenes. Applied Food Biotechnology. 2015;2(4):11-9. doi:doi.org/10.22037/afb.v2i4.8894.

8. Yeganeh M, Hosseini H, Mehrabian S, Torbati ES, Zamir SM. Antibiofilm Effects of Lactobacilli against Ciprofloxacin-Resistant Uropathogenic Escherichia coli strains in Pasteurized Milk. Applied Food Biotechnology. 2017;4(4):241-50. doi:doi.org/10.22037/afb.v4i4.17783.

9. Ahmadi Badi S, Moshiri A, Fateh A, Rahimi Jamnani F, Sarshar M, Vaziri F et al. Microbiota-Derived Extracellular Vesicles as New Systemic Regulators. Frontiers in Microbiology. 2017;8:1610. doi:doi.org/10.3389/fmicb.2017 .01610 .

10. Brown L, Wolf JM, Prados-Rosales R, Casadevall A. Through the wall: extracellular vesicles in Gram-positive bacteria, mycobacteria and fungi. Nature Reviews Microbiology. 2015;13(10):620. doi:doi:10.1038/nrmicro34 80 .

11. Ebrahimi Vargoorani M, Modarressi MH, Sheikhpour M, Siadat SD. Medical Function of Bacterial Extracellular Vesicles. Iranian Journal of Medical Microbiology. 2018;12(3):140-59. doi:10.30699/ijmm.12.3.140.

12. Kim S-H, Kim K-S, Lee S-R, Kim E, Kim M-S, Lee E-Y et al. Structural modifications of outer membrane vesicles to refine them as vaccine delivery vehicles. Biochimica et Biophysica Acta (BBA) Biomembranes. 2009;1788(10):215 0-9.

13. Mathivanan S, Lim JW, Tauro BJ, Ji H, Moritz RL, Simpson RJ. Proteomics analysis of A33 immunoaffinitypurified exosomes released from the human colon tumor cell line LIM1215 reveals a tissue-specific protein signature. Molecular \& Cellular Proteomics. 2010;9(2):197-208. doi:doi: 10.1074/mcp.M900152-MCP200.

14. Taylor DD, Shah S. Methods of isolating extracellular vesicles impact down-stream analyses of their cargoes. Methods. 2015;87:3-10.

15. Lane RE, Korbie D, Anderson W, Vaidyanathan R, Trau M. Analysis of exosome purification methods using a model liposome system and tunable-resistive pulse sensing. Scientific reports. 2015;5:7639. doi: doi:10.1038/srep07639. 16. Rider MA, Hurwitz SN, Meckes Jr DG. ExtraPEG: a polyethylene glycol-based method for enrichment of extracellular vesicles. Scientific reports. 2016;6:23978.

17. Xu M, Gagné-Bourque F, Dumont M-J, Jabaji S. Encapsulation of Lactobacillus casei ATCC 393 cells and evaluation of their survival after freeze-drying, storage and under gastrointestinal conditions. Journal of Food Engineering. 2016;168:52-9.

18. Nakao R, Hasegawa H, Ochiai K, Takashiba S, Ainai A, Ohnishi $M$ et al. Outer membrane vesicles of Porphyromonas gingivalis elicit a mucosal immune response. PloS one. 2011;6(10):e26163.

19. Brown L, Kessler A, Cabezas-Sanchez P, Luque-Garcia JL, Casadevall A. Extracellular vesicles produced by the Gram-positive bacterium Bacillus subtilis are disrupted by the lipopeptide surfactin. Molecular microbiology. 2014;93(1):183-98. doi:doi.org/10.1111/mmi.12650.

20. Van der Pol E, Böing A, Gool E, Nieuwland R. Recent 
developments in the nomenclature, presence, isolation, detection and clinical impact of extracellular vesicles. Journal of Thrombosis and Haemostasis. 2016;14(1):48-56. 21. Gould SJ, Raposo G. As we wait: coping with an imperfect nomenclature for extracellular vesicles. Journal of extracellular vesicles. 2013;2(1):20389.

22. Lötvall J, Hill AF, Hochberg F, Buzás EI, Di Vizio D, Gardiner $\mathrm{C}$ et al. Minimal experimental requirements for definition of extracellular vesicles and their functions: a position statement from the International Society for Extracellular Vesicles. Taylor \& Francis; 2014.

23. Gardiner C, Vizio DD, Sahoo S, Théry C, Witwer KW, Wauben $\mathrm{M}$ et al. Techniques used for the isolation and characterization of extracellular vesicles: results of a worldwide survey. Journal of extracellular vesicles. 2016;5(1):32945.

24. Lee EY, Choi DY, Kim DK, Kim JW, Park JO, Kim S et al. Gram-positive bacteria produce membrane vesicles: proteomics-based characterization of Staphylococcus aureus-derived membrane vesicles. Proteomics. 2009;9(24):5425-36.

25. Haas B, Grenier D. Isolation, characterization and biological properties of membrane vesicles produced by the swine pathogen Streptococcus suis. PLoS One. 2015;10(6):e0130528.

26. Brown L, Kessler A, Cabezas-Sanchez P, Luque-Garcia JL, Casadevall A. Extracellular vesicles produced by the $\mathrm{G}$ ram-positive bacterium $B$ acillus subtilis are disrupted by the lipopeptide surfactin. Molecular microbiology. 2014;93(1):183-98.
27. Dean SN, Leary DH, Sullivan CJ, Oh E, Walper SA. Isolation and characterization of Lactobacillus-derived membrane vesicles. Scientific reports. 2019;9(1):877.

28. Deregibus MC, Figliolini F, D'antico S, Manzini PM, Pasquino $\mathrm{C}$, De Lena $\mathrm{M}$ et al. Charge-based precipitation of extracellular vesicles. International journal of molecular medicine. 2016;38(5):1359-66. doi:doi.org/10.3892/ijmm.20 16.2759 .

29. Niu Z, Pang RT, Liu W, Li Q, Cheng R, Yeung WS. Polymer-based precipitation preserves biological activities of extracellular vesicles from an endometrial cell line. PloS one. 2017;12(10):e0186534.

30. Choi SJ, Kim M-H, Jeon J, Kim OY, Choi Y, Seo J et al. Active immunization with extracellular vesicles derived from Staphylococcus aureus effectively protects against staphylococcal lung infections, mainly via Th1 cellmediated immunity. PloS one. 2015;10(9):e0136021.

31. Jiang Y, Kong Q, Roland KL, Curtiss III R. Membrane vesicles of Clostridium perfringens type A strains induce innate and adaptive immunity. International journal of medical microbiology. 2014;304(3-4):431-43.

32. Olaya-Abril A, Prados-Rosales R, McConnell MJ, Martín-Peña R, González-Reyes JA, Jiménez-Munguía I et al. Characterization of protective extracellular membranederived vesicles produced by Streptococcus pneumoniae. Journal of proteomics. 2014;106:46-60.

33. Akhter N, Wu B, Memon AM, Mohsin M. Probiotics and prebiotics associated with aquaculture: a review. Fish \& shellfish immunology. 2015;45(2):733-41. 